\title{
365 Patients with Pectus Excavatum Undergoing Video-Assisted Thoracoscopic Surgery for the Nuss Procedure: A 5-Year Single-Institution Experience
}

Nguyen The May, M.D. ( $\sim$ nguyenthemay@gmail.com )

Viet-Tiep Friendship Hospital, Hai Phong 180000, Vietnam

Nguyen Huu Uoc, M.D., Ph.D.

Department of Cardiovascular and Thoracic Surgery, Viet Duc University Hospital, Hanoi 100000, Vietnam https://orcid.org/0000-0002-6981-9837

Pham Huu Lu, M.D., Ph.D.

Department of Cardiovascular and Thoracic Surgery, Viet Duc University Hospital, Hanoi 100000, Vietnam

Phung Duy Hong Son, M.D., Ph.D.

Department of Cardiovascular and Thoracic Surgery, Viet Duc University Hospital, Hanoi 100000, Vietnam

Vu Ngoc Tu, M.D., Ph.D.

Department of Cardiovascular and Thoracic Surgery, Viet Duc University Hospital, Hanoi 100000, Vietnam https://orcid.org/0000-0003-4262-4471

Nguyen Viet Anh, M.D.

Department of Cardiovascular and Thoracic Surgery, Viet Duc University Hospital, Hanoi 100000, Vietnam

Khanh Nam Do, M.Sc.

Institute for Preventive Medicine and Public Health, Hanoi Medical University, Hanoi 100000, Vietnam

Kim-Duy Vu

Institute for Preventive Medicine and Public Health, Hanoi Medical University, Hanoi 100000, Vietnam

Hoang-Long Vo, M.D. ( $\nabla$ vohoanglonghmu@gmail.com )

Institute for Preventive Medicine and Public Health, Hanoi Medical University, Hanoi 100000, Vietnam https://orcid.org/0000-0001-5992-875X

Doan Quoc Hung, M.D., Ph.D. ( $\nabla$ hung.doanquoc@gmail.com )

Hanoi Medical University, Hanoi 100000, Vietnam

\section{Research Article}

Keywords: Pectus excavatum, Nuss procedure, Thoracoscopic surgery, VATS-NUSS 
Posted Date: September 2nd, 2020

DOI: https://doi.org/10.21203/rs.3.rs-67075/v1

License: (c) (i) This work is licensed under a Creative Commons Attribution 4.0 International License. Read Full License 


\section{Abstract}

Objective: We conducted this study to review our large single-center experience in Vietnam in the use of the VATS-NUSS for 365 consecutive patients with PE within the five years and to evaluate mid-term to long-term outcome in these patients.

Methods: In this retrospective study, we consecutively selected the patients with the diagnosis of PE who underwent VATS-NUSS from January 2015 to September 2019. A total of 365 patients who were surgically treated for PE at the Department of Cardiovascular and Thoracic Surgery, Viet Duc University Hospital (Hanoi, Vietnam) was included in final analysis.

Results: The length of postoperative hospitalization ranged from 1 to 13 days (Mean: 5.1). Early postoperative complications were recorded up to 30 days during hospital stay, consisting of pneumothorax $(n=5,1.37 \%)$, pleural bleeding / pleural fluid $(n=2,0.55 \%)$, pleural hematoma $(n=1$, $0.27 \%$ ), pneumonia $(n=1,0.27 \%)$, surgical wound infection $(n=1,0.27 \%)$, incision fluid accumulation ( $n$ $=3,0.82 \%)$, metal bar infection $(n=1,0.27 \%)$, atelectasis $(n=3,0.82 \%)$, and fever $(n=8,2.19 \%)$. In the late postoperative complications, surgical wound infection $(n=2,0.55 \%)$, metal bar deviation $(n=5$, $1.37 \%)$, metal bar allergy $(n=10,2.74 \%)$, recurrent $\operatorname{PE}(n=2,0.55 \%)$, and persistent $P E(n=5,1.37 \%)$ were observed. No deaths occurred. The outcomes related to the bar removal that was carried out in 175 patients (47.95\%). The time the bar was in situ ranged from 2 to 49 months (mean: $28.89 \pm 7.48$ years). The mean operative time at bar removal was $34.09 \pm 10.61$ minutes, and the length of hospitalization following bar removal was $2.4 \pm 1.34$ days. Of 175 patients performed bar removal, the most frequent complication was pneumothorax, which was diagnosed in 19 (10.85\%) patients; surgical wound infection in 1 patient and incision fluid accumulation in 1 patient were observed following bar removal. Towards mid-term results at $6-30$ months postoperatively, there were 124 out of 365 patients (33.97\%). Of 124 patients, most patients were unremoved the bar $(n=121,97.58 \%)$, and only 3 patients were removed the bar (2.42\%). Most patients had body weight gain $(n=97,78.23 \%)$, and increased physical activity and improved health $(n=115,92.74 \%)$. The Haller index on chest X-ray was $2.44 \pm 0.15 .220$ out of 365 patients (60.27\%) were postoperatively evaluated long-term results at over 30 months. Of those, 172 received bar removal (78.18\%) and 48 have not yet received bar removal (21.82\%). 217 patients (98.64\%) had both body weight gain, and increased physical activity and improved health. The Haller index on chest X-ray was $2.45 \pm 0.21$.

Conclusions: The VATS-NUSS for PE was safe and effective approach with minimizing the occurrence of serious intra- and postoperative complications. From our initial one-institution experience in a resourcescare country, good mid-term to long-term postoperative outcomes in PE patients were obtained with VATS-NUSS. Current rare evidence drawn from this cohort enables to give a real picture in the application, modification and development of VATS-NUSS not only for Vietnam but also the countries having similar resource-scare conditions. 


\section{Introduction}

Since Nuss et al.'s report with 42 patients using a minimally invasive technique to correct the depression of the pectus excavatum (PE) in 1998 [1], this procedure (Nuss procedure) was considered the gold standard treatment for PE in prepubertal patients. The initial application of the Nuss technique was relatively limited; however, thanks to accumulated experiences across the world, the Nuss technique with several modifications has been used extensively by many surgeons, such as adults [2], severe and/or asymmetrical deformities [3], and recurrent cases [4], [5], [6], [7]. In particular, the relative risk of complications significantly decreased, such as bar rotation, pneumothorax, and severe cardiopulmonary injury [5]. Various attempts have recently been made to reduce the risk of severe complications, including utilizing the originally designed sternum elevator or bar insertion with the extrathoracic approach [8], [9].

With universal acceptance, the additional use of the video-assisted thoracoscopic surgery (VATS) technique in the Nuss procedure demonstrated an increased safety of the operation with a decreased frequency of serious intraoperative and postoperative complications. Bufo et al. first reported VATS for the prevention of life-threatening intraoperative lesions of the mediastinum [10]. The discussion is currently focused on the superiority of left, right or bilateral thoracoscopic access and the necessity of CO2 insufflation or sternal elevation to improve safety during a surgical procedure [11], [12], [13], [14]. Although serious life-threatening complications after the Nuss procedure are rare [15], [16], [17], VATS used during the operation may further reduce the perioperative risk. The available evidence in the literature was mostly reported from large institutions in developed countries, and no publications on the surgical outcomes and safety in the application of VATS for the Nuss procedure were known in resourcescare conditions such as Vietnam.

Therefore, this study was conducted to review our large single-center experience in Vietnam in the use of the VATS technique in the Nuss procedure for 365 consecutive patients with PE within the five years and to evaluate mid-term to long-term outcomes in these patients.

\section{Methods}

\section{Patients and Methods}

\section{Study Population}

In this retrospective study, we consecutively selected patients with a diagnosis of PE who underwent VATS for the NUSS procedure from January 2015 to September 2019. A total of 365 patients who were surgically treated for PE at the Department of Cardiovascular and Thoracic Surgery, Viet Duc University Hospital (Hanoi, Vietnam) were included in the final analysis.

The evaluation by complete history, physical examination, chest radiographs, electrocardiogram, pulmonary function test, echocardiogram, and computerized tomography (CT) of the chest were performed. The demographic data, surgical data, thoracoscopic findings, early and late complications, 
length of hospital stay, and early, mid-term, and long-term outcomes were also collected. The indications for surgical repair were two or more of the following criteria demonstrated by Dr Nuss: (1) progression of the deformity; (2) exercise intolerance; (3) progressive chest pain or dyspnea; (4) restrictive ventilatory impairment; (5) Haller index > 3.25; (6) previous failed Ravitch procedure; (7) cardiac compression; and (8) mitral valve prolapse. All procedures were performed with the Nuss procedure, which included left, right or bilateral VATS.

\section{Surgical techniques}

All patients had a thoracic epidural for intraoperative anesthesia and postoperative pain control. The patient was placed in the supine position after general anesthesia. The arms of the patient were kept abducted approximately $70-80^{\circ}$ in relation to the body (Figure 1 ).

In the determination of anatomic landmarks in surgery, we determined the concave area, the center of the concave area, the edges of the concave area, and the highest point of the concave edge on either side of the concave circumference. The metal bar was placed along a straight line that was formed from the center of the concave area and the two highest points of the concave edge (Figure 2). The measurements of the thorax and the bending of the metal bar (sternum lift) were then performed (Figure 3).

A 5-mm trocar was placed in the marked position on the left chest wall. After pumping low-pressure CO2 $(5 \mathrm{mmHg})$ into the pleural cavity to collapse the lungs, the camera with a 30-degree optic was taken to the left chest to observe the entire chest and mediastinum during surgery. The skin incision was made above or below the position where the metal rod was intended to be placed in an intercostal space. One 2-cm vertical skin incision was made in the midaxillary line on each side (Figure 4). In the required cases with 2-bar insertion, only a skin incision was made between the two intended positions to place the two metal bars. Subcutaneous or submuscular dissections were performed from the cutaneous incision to the highest edge of the PE (Figure 5).

Then, we created a tunnel through the mediastinum (Figure 6). The heart-shaped clamp was used to pierce the left pleural space at the highest edge of the pit, slowly went close to the anterior chest wall towards the mediastinum at the deepest point of the pit, and gradually separated the pericardium from the posterior sternum. The introducer reaches the posterior surface of the sternum in the left pleural cavity and is further moved through the mediastinal pleura in the retrosternal space, next goes across the right pleural space and finally exits the chest through the right intercostal space. The electrocardiogram is continuously monitored during the process of creating a tunnel through the mediastinum.

During the reeving sternum lift bar stage, a Perlon thread was tied to the metal rod and then tied to the introducer. After the withdrawn introducer was withdrawn, the metal bar was threaded through the anterior mediastinum in a direction going from right to left under the control of thoracoscopy (Figure 7). Then, the pectus bar was rotated to suit the patient's chest (Figure 8). 
The steel thread was sewn around the ribs and was then tied to the top of the metal bar (Figure 9). Under the observation of endoscopy, the process of sewing steel threads was very safe, avoiding stitching into the lungs and checking bleeding. We did not use screw braces to fix the metal bar as in the original Nuss surgery. Finally, the anesthesiologist squeezed the balloon through the endotracheal tube to allow the expanded lungs while gradually withdrawing the trocar and removing air from the bilateral pleural space. A pleural drainage catheter was not placed.

\section{Statistical Analysis}

We conducted all analyses with Stata ${ }^{\circledR} 15$ (StataCorp LLC, USA) for Windows. Continuous variables were expressed as the mean and standard deviation (SD) with interquartile ranges (IQR). Categorical variables are presented as counts with percentages. No imputation was made for missing data. A $p$-value $<0.05$ was considered to be statistically significant.

\section{Ethical Approval}

All procedures conducted in studies involving human subjects were in compliance with the ethical principles of the Institutional and/or National Study Committee and the Helsinki Declaration of 1964 and its corresponding amendments or equivalent ethical standards. This study was approved by the Ethics Board of Hanoi Medical University. All individual participants included in the study obtained informed consent.

\section{Results}

\section{Preoperative clinical characteristics of PE patients who underwent VATS-NUSS}

Table 1 shows the preoperative clinical characteristics of 365 PE patients undergoing VATS for the NUSS procedure. The median age at operation was $15.61 \pm 3.73$ years (range, 5 to 27 years). A total of $84.65 \%$ were male. PE was commonly detected at puberty $(n=328,89.9 \%)$. Underweight $(B M I<18.5)$ was found in $84.4 \%$ of the patients, and no patient was recorded as overweight or obese. A total of $1.37 \%$ of the patients experienced previous PE repair surgery $(n=5)$. Of the 365 patients, 288 had symmetric PE (78.90\%), and 77 had asymmetric PE (21.10\%). Additionally, in Table 1, the numeric distribution of each morphologic type and subtype of pectus is described in detail.

Palpitations were present in $81.64 \%$ of the patients $(n=298)$. The other common symptoms were inferiority complex $(n=262,71.78 \%)$, dyspnea on exertion $(n=218,59.73 \%)$, shortness of breath while exercising ( $n=203,55.62 \%)$, development of poor physical health $(n=192,52.60 \%)$, and chest pain while exercising $(n=149,40.82 \%)$; dsementia was uncommon $(n=3,0.82 \%)$ (Table 1$)$.

\section{Table 1. Preoperative clinical characteristics of patients $(n=365)$}




\begin{tabular}{|c|c|}
\hline Characteristics & $\begin{array}{l}\text { Patients } \\
(\mathrm{n}=365)\end{array}$ \\
\hline \multicolumn{2}{|l|}{ Age } \\
\hline Mean \pm SD - years & $15.61 \pm 3.73$ \\
\hline Range - years & $5-27$ \\
\hline Male sex - no. (\%) & 309 (84.65) \\
\hline \multicolumn{2}{|l|}{ BMI - no. (\%) } \\
\hline Underweight (BMI <18.5) & $308(84.4)$ \\
\hline Normal $(18.5 \leq$ BMI <23.0) & $57(15.6)$ \\
\hline Overweight and obesity (BMI 23 ) & $0(0.0)$ \\
\hline \multicolumn{2}{|l|}{ Time PE was detected - no. (\%) } \\
\hline At birth & $11(3.0 \%)$ \\
\hline Childhood & $26(7.1 \%)$ \\
\hline Puberty & $328(89.9 \%)$ \\
\hline Previous PE repair surgery - no. (\%) & $5(1.37)$ \\
\hline Family history of PE - no. (\%) & $46(12,60)$ \\
\hline \multicolumn{2}{|l|}{ Comorbidity - no. (\%) } \\
\hline Prolonged respiratory tract inflammation & $2(0.55)$ \\
\hline Asthma & $1(0.27)$ \\
\hline Congenital heart disease & $22(6,03)$ \\
\hline Scoliosis & $1(0.27)$ \\
\hline Dementia & $3(0.82)$ \\
\hline Marfan syndrome & $1(0.27)$ \\
\hline \multicolumn{2}{|l|}{ PE defect type - no. (\%) } \\
\hline Bilateral symmetry & $288(78.90)$ \\
\hline Asymmetry to the right & $70(19.18)$ \\
\hline Asymmetry to the left & $7(1.92)$ \\
\hline \multicolumn{2}{|l|}{ Morphologic Classification ${ }^{\mathrm{a}}-$ no. (\%) } \\
\hline Type 1A: prototype & $252(69.04)$ \\
\hline Type 1B: broad-flat & $70(19.18)$ \\
\hline \multicolumn{2}{|l|}{ Type 2A: eccentric } \\
\hline Type 2A1: focal & $14(3.84)$ \\
\hline Type 2A2: broad-flat & $3(0.82)$ \\
\hline Type 2A3: long canal & $1(0.27)$ \\
\hline Type 2B: unbalanced & $24(6.58)$ \\
\hline Type 2C: combined & $1(0.27)$ \\
\hline \multicolumn{2}{|l|}{ Clinical symptoms } \\
\hline Inferiority complex - no. (\%) & $262(71,78)$ \\
\hline Chest pain while exercising - no. (\%) & $149(40.82)$ \\
\hline Dyspnea on exertion - no. (\%) & $218(59.73)$ \\
\hline Shortness of breath while exercising - no. (\%) & $203(55.62)$ \\
\hline Palpitations - no. (\%) & $298(81.64)$ \\
\hline Development of poor physical health - no. (\%) & $192(52.6)$ \\
\hline Dsementia - no. (\%) & $3(0.82)$ \\
\hline
\end{tabular}

${ }^{a}$ Morphologic classification by Hyung Joo Park [18] 


\section{Preoperative subclinical characteristics of PE patients who underwent VATS-NUSS}

The preoperative subclinical characteristics of 365 PE patients undergoing VATS for the NUSS procedure are summarized in Table 2. ECG abnormalities were documented in $41.10 \%(n=150)$; the most common abnormality on ECG was sinus tachycardia $(n=90,26.66 \%)$; increased right ventricular mass was present in $9.04 \%$ of the patients $(n=33)$, RBBB in $4.93 \%(n=18)$, LBBB in $1.10 \%(n=4)$, ectopic rhythm in $1.37 \%$ $(n=5)$, sinus bradycardia in $0.27 \%(n=1)$, increased left ventricular mass in $0.27 \%(n=1)$, and atrial fibrillation in $0.27 \%(n=1)$. On Doppler echocardiography, $6.58 \%$ of patients had tricuspid valve regurgitation $(n=24)$, while the figure for mitral valve regurgitation was $3.84 \%(n=14)$; PFO was detected among 22 patients $(6.03 \%)$; and elevated PAP was detected in 33 patients $(3.94 \%)$. The mean ejection fraction (EF) was $67.39 \pm 5.91 \%$. In 365 patients with PE presenting for surgical treatment, the mean FVC was $63.80 \pm 11.26$, the mean $\mathrm{FEV}_{1}$ was $70.69 \pm 11.8 \%$, the mean $\mathrm{FEV}_{1} / \mathrm{FVC}$ was $111.91 \pm 9.45$, and the mean $\mathrm{FEF}_{25}$

Table 2. Preoperative Subclinical Characteristics of Patients $(n=365)$ 


\begin{tabular}{|c|c|}
\hline Characteristics & $\begin{array}{c}\text { Patients } \\
(\mathrm{n}=365) \\
\end{array}$ \\
\hline Electrocardiogram abnormalities - no. (\%) & $150(41.10)$ \\
\hline Sinus tachycardia - no. (\%) & $90(24.66)$ \\
\hline Sinus bradycardia - no. (\%) & $1(0.27)$ \\
\hline Right bundle branch block (RBBB) - no. (\%) & $18(4.93)$ \\
\hline Left bundle branch block (LBBB) - no. (\%) & $4(1.10)$ \\
\hline Increased right ventricular mass - no. (\%) & $33(9,04)$ \\
\hline Increased left ventricular mass - no. (\%) & $1(0,27)$ \\
\hline Ectopic rhythm - no. (\%) & $5(1,37)$ \\
\hline Atrial fibrillation - no. (\%) & $1(0,27)$ \\
\hline \multicolumn{2}{|l|}{ Doppler echocardiography results } \\
\hline Tricuspid valve regurgitation - no. (\%) & $24(6.58)$ \\
\hline Mitral valve regurgitation - no. (\%) & $14(3.84)$ \\
\hline $\mathrm{PFO}-$ no. (\%) & $22(6.03)$ \\
\hline Elevated PAP - no. (\%) & $33(9.04)$ \\
\hline \multicolumn{2}{|l|}{$\mathrm{EF}(\mathrm{n}=365)$} \\
\hline Mean \pm SD - \% & $67.39 \pm 5.91$ \\
\hline Range - \% & $56-79.51$ \\
\hline \multicolumn{2}{|l|}{ Pulmonary function tests } \\
\hline FVC - Mean \pm SD & $63.80 \pm 11.26$ \\
\hline $\mathrm{FEV}_{1} \%-\mathrm{Mean} \pm \mathrm{SD}$ & $70.69 \pm 11.8$ \\
\hline $\mathrm{FEV}_{1} / \mathrm{FVC}-\mathrm{Mean} \pm \mathrm{SD}$ & $111.91 \pm 9.45$ \\
\hline $\mathrm{FEF}_{25-75 \%}-\mathrm{Mean} \pm \mathrm{SD}$ & $98.62 \pm 23.65$ \\
\hline \multicolumn{2}{|l|}{ Classification of pulmonary function test value } \\
\hline FVC $<80 \%-$ no. $(\%)$ & $334(93.56 \%)$ \\
\hline FEV1< $80 \%-$ no. $(\%)$ & $277(77.59 \%)$ \\
\hline FEV1/FVC $<70 \%-$ no. (\%) & $2(0.57 \%)$ \\
\hline $\mathrm{FEF}_{25-75 \%}<60 \%-$ no. $(\%)$ & $12(3.34 \%)$ \\
\hline \multicolumn{2}{|l|}{ Chest CT scan } \\
\hline Twisting the sternum - no. (\%) & 79 (21.64) \\
\hline Scoliosis - no. (\%) & $3(0.82)$ \\
\hline Heart compression, the heart pushes to the left - no. (\%) & $58(15,89)$ \\
\hline Bronchiectasis - no. (\%) & $2(0.55)$ \\
\hline \multicolumn{2}{|l|}{ Haller index measuring on X-ray and CT scan } \\
\hline \multicolumn{2}{|l|}{ Haller index on chest CT scan $(\mathrm{n}=365)$} \\
\hline Mean \pm SD & $3.81 \pm 0.78$ \\
\hline Range & $2.58-14.5$ \\
\hline \multicolumn{2}{|l|}{ Haller index on chest X-ray $(n=365)$} \\
\hline Mean \pm SD & $3.83 \pm 0.98$ \\
\hline Range & $2.56-14.5$ \\
\hline
\end{tabular}

SD: standard deviation;RBBB: right bundle branch block; LBBB; left bundle branch block; PFO: patent foramen ovale; PAP:pulmonary artery pressure; CT: computed tomography Operative characteristics of PE patients who underwent VATS-NUSS

One bar was indicated for most patients ( $n=350,95.89 \%)$, and two bars were required in 15 patients (4.11\%). The mean operative time was 49.54 minutes. A total of $85.48 \%$ of the patients had a left 
operative wound from the left to the right pleural cavity $(n=312)$, while $14.52 \%$ had a left to the right pleural cavity $(n=53)$. Steel sutures on the left side combined with Vicryl sutures on the right side were commonly utilized to fix metal corrective bars $(n=320,87.67 \%)$. Epidural anesthesia was used for postoperative

Table 3. Operative Characteristics of Patients $(n=365)$

\begin{tabular}{lc}
\hline \multicolumn{1}{c}{ Characteristics } & $\begin{array}{c}\text { Patients } \\
(\mathbf{n}=365)\end{array}$ \\
\hline Number of bars - no. (\%) & $350(95.89)$ \\
One bar & $15(4.11)$ \\
Two bars & \\
VATS side - no. (\%) & $308(84.38)$ \\
Left VATS & $43(11.78)$ \\
Right VATS & $14(3.84)$ \\
Bilateral VATS & \\
The steel introducer - no. (\%) & $312(85.48)$ \\
Left-to-right & $53(14.52)$ \\
Right-to-left & $320(87.67)$ \\
Method of metal corrective bar fixation - no. (\%) & $34(9.32)$ \\
$\quad$ Steel sutures (left) + Vicryl sutures (right) & $10(2.74)$ \\
Steel sutures (right) + Vicryl sutures (left) & $1(0.27)$ \\
Bilateral steel sutures & $278(76.16)$ \\
Bilateral Vicryl sutures & $1(0.27)$ \\
Postoperative pain relief by epidural anesthesia - no. (\%) & \\
Accidents in surgery - no. (\%) & 49.54 \\
Operative time & $20-140$ \\
$\quad$ Mean \pm SD - Mins & \\
Range - Mins &
\end{tabular}

SD: standard deviation

\section{Postoperative characteristics of PE patients who underwent VATS-NUSS}

The length of postoperative hospitalization ranged from 1 to 13 days (Mean: 5.1). Early postoperative complications were recorded up to 30 days during the hospital stay. These events largely consisted of pneumothorax $(n=5,1.37 \%)$, pleural bleeding/pleural fluid $(n=2,0.55 \%)$, pleural hematoma $(n=1$, $0.27 \%$ ), pneumonia $(n=1,0.27 \%)$, surgical wound infection $(n=1,0.27 \%)$, incision fluid accumulation ( $n$ $=3,0.82 \%)$, metal bar infection $(n=1,0.27 \%)$, atelectasis $(n=3,0.82 \%)$, and fever $(n=8,2.19 \%)$. In the late postoperative complications, surgical wound infection $(n=2,0.55 \%)$, metal bar deviation $(n=5$, $1.37 \%)$, metal bar allergy $(n=10,2.74 \%)$, recurrent PE $(n=2,0.55 \%)$, and persistent $P E(n=5,1.37 \%)$ were observed. No deaths occurred (Table 4). 
Table 4 also indicates the outcomes related to the bar removal that was carried out in 175 patients (47.95\%). The time the bar was in situ ranged from 2 to 49 months (mean: $28.89 \pm 7.48$ years). In 3 patients $(1.71 \%)$, bar removal was performed early, less than 1 year, due to metal bar allergy. We recorded a mean operative time at bar removal of $34.09 \pm 10.61$ minutes and a length of hospitalization following bar removal of $2.4 \pm 1.34$ days. Of 175 patients who underwent bar removal, the most frequent complication was pneumothorax, which was diagnosed in 19 (10.85\%) patients; surgical wound infection in 1 patient and incision fluid accumulation in 1 patient were observed following bar removal.

Table 4. Postoperative Data of Patients 


\begin{tabular}{|c|c|}
\hline & $\begin{array}{l}\text { Patients } \\
(\mathrm{n}=365)\end{array}$ \\
\hline \multicolumn{2}{|l|}{ Length of postoperative hospitalization } \\
\hline Mean \pm SD - days & 5.1 \\
\hline Range - days & $1-13$ \\
\hline \multicolumn{2}{|l|}{ Early postoperative complications } \\
\hline Pneumothorax - no. (\%) & $5(1.37)$ \\
\hline Pleural bleeding / pleural fluid - no. (\%) & $2(0.55)$ \\
\hline Pleural hematoma - no. (\%) & $1(0,27)$ \\
\hline Pneumonia - no. (\%) & $1(0.27)$ \\
\hline Surgical wound infection - no. (\%) & $1(0.27)$ \\
\hline Incision fluid accumulation - no. (\%) & $3(0.82)$ \\
\hline Metal bar infection - no. (\%) & $1(0.27)$ \\
\hline Atelectasis - no. (\%) & $3(0.82)$ \\
\hline Fever - no. (\%) & $8(2.19)$ \\
\hline \multicolumn{2}{|l|}{ Late postoperative complications } \\
\hline Surgical wound infection - no. (\%) & $2(0.55)$ \\
\hline Metal bar deviation - no. (\%) & $5(1.37)$ \\
\hline Metal bar allergy - no. (\%) & $10(2,74)$ \\
\hline Recurrent PE - no. (\%) & $2(0.55)$ \\
\hline \multirow[t]{2}{*}{ Persistent PE - no. (\%) } & $5(1.37)$ \\
\hline & $\begin{array}{c}\text { Patients } \\
(\mathrm{n}=175)\end{array}$ \\
\hline \multicolumn{2}{|l|}{ Time the bar was in situ - no. (\%) } \\
\hline$<1$ - years & $3(1.71)$ \\
\hline $1 £-<2-$ years & $16(9.14)$ \\
\hline $2 £-<3-$ years & $103(58.86)$ \\
\hline $3 £-<4-$ years & $49(28.00)$ \\
\hline$\square 4$ - years & 4 (2.29) \\
\hline Mean \pm SD - months & $28.89 \pm 7.48$ \\
\hline Range - months & $2-49$ \\
\hline \multicolumn{2}{|l|}{ Operative time at bar removal } \\
\hline Mean \pm SD - mins & $34.09 \pm 10.61$ \\
\hline Range - mins & $20-110$ \\
\hline \multicolumn{2}{|l|}{ Length of hospitalization following bar removal } \\
\hline Mean \pm SD - days & $2.4 \pm 1.34$ \\
\hline Range - days & $1-14$ \\
\hline \multicolumn{2}{|l|}{ Complications at bar removal } \\
\hline Pneumothorax - no. (\%) & $19(10.85)$ \\
\hline Surgical wound infection - no. (\%) & $1(0.57)$ \\
\hline Incision fluid accumulation - no. (\%) & $1(0.57)$ \\
\hline
\end{tabular}

\section{Mid-term to long-term outcome in PE patients undergoing VATS-NUSS}

Towards mid-term results at $6-30$ months postoperatively, there were 124 out of 365 patients (33.97\%). Of 124 patients, most patients had unremoved bars $(n=121,97.58 \%)$, and only 3 patients had removed bars $(2.42 \%)$. Most patients had body weight gain $(n=97,78.23 \%)$, increased physical activity and improved health $\left(n=115,92.74 \%\right.$ ). In 124 patients, the mean BMI index was $18.77 \pm 0.92 \mathrm{~kg} / \mathrm{m}^{2}$, and the Haller index on chest X-ray was $2.44 \pm 0.15$. Overall, the patients receiving bar removal had a significantly 
higher mean BMI index compared to those not yet receiving bar removal (20.30 \pm 1.11 vs $18.73 \pm 0.89)$ ( $p$ $<0.05)$. The proportion of patients with increased physical activity and improved health following surgery differed significantly between those receiving bar removal $(n=43,100.00 \%)$ and those not yet receiving bar removal $(n=112,92.56 \%)(p<0.05)($ Table 5).

Postoperative long-term results at over 30 months are also indicated in Table 5. A total of 220 out of 365 patients (60.27\%) were included in this phase. Of those, 172 received bar removal $(78.18 \%)$, and 48 had not yet received bar removal (21.82\%). A total of 217 patients $(98.64 \%)$ had both body weight gain, increased physical activity and improved health. In 220 patients over 30 months postoperatively, the mean BMI index was $19.02 \pm 0.99 \mathrm{~kg} / \mathrm{m}^{2}$, and the Haller index on chest X-ray was $2.45 \pm 0.21$. Overall, the patients receiving bar removal had a significantly higher mean BMI index compared to those not yet receiving bar removal $(19.13 \pm 1.01$ vs $18.62 \pm 0.82)(p<0.05)$.

Table 5. Mid-term to long-term postoperative outcome in PE patients

Mid-term postoperative outcome $(\mathrm{n}=124)$

(6-30 months postoperatively)

\begin{tabular}{|c|c|c|c|c|}
\hline & \multirow{2}{*}{$\begin{array}{l}\text { All patients } \\
(\mathrm{n}=124)\end{array}$} & \multicolumn{2}{|c|}{ Bar removal } & \multirow{3}{*}{$\mathrm{p}$ value } \\
\hline & & Yes $(n=3)$ & No $(n=121)$ & \\
\hline & $\begin{array}{l}\text { Count (\% of } \\
\text { total) }\end{array}$ & $\begin{array}{l}\text { Count }(\% \text { of } \\
\text { total) }\end{array}$ & $\begin{array}{l}\text { Count }(\% \text { of } \\
\text { total) }\end{array}$ & \\
\hline Body weight gain & $97(78.23)$ & $3(100.00)$ & $94(77.69)$ & $1.000^{*}$ \\
\hline \multirow{2}{*}{$\begin{array}{l}\text { Increased physical activity and } \\
\text { improved health }\end{array}$} & $115(92.74)$ & $3(100.00)$ & $112(92.56)$ & \multirow[t]{2}{*}{$<0.05^{*}$} \\
\hline & Mean (SD) & Mean (SD) & Mean (SD) & \\
\hline BMI index - kg/m $]$ & $18.77 \pm 0.92$ & $20.30 \pm 1.11$ & $18.73 \pm 0.89$ & $<0.05^{* *}$ \\
\hline Haller index on chest X-ray & $2.44 \pm 0.15$ & $2.5 \pm 0.17$ & $2.44 \pm 0.15$ & $0.382^{* *}$ \\
\hline \multicolumn{5}{|c|}{$\begin{array}{c}\text { Long-term postoperative outcome }(\mathrm{n}=220) \\
\text { (over } 30 \text { months postoperatively) }\end{array}$} \\
\hline & \multirow{2}{*}{$\begin{array}{l}\text { All patients } \\
(\mathrm{n}=220)\end{array}$} & \multicolumn{2}{|c|}{ Bar removal } & \multirow[t]{3}{*}{$\mathrm{p}$ value } \\
\hline & & Yes $(n=172)$ & No $(n=48)$ & \\
\hline & $\begin{array}{l}\text { Count (\% of } \\
\text { total) }\end{array}$ & $\begin{array}{l}\text { Count (\% of } \\
\text { total) }\end{array}$ & $\begin{array}{l}\text { Count (\% of } \\
\text { total) }\end{array}$ & \\
\hline Weight gain & $217(98.64)$ & $171(99.42)$ & $46(95.83)$ & $0.321^{*}$ \\
\hline \multirow{2}{*}{$\begin{array}{l}\text { Increased physical activity and } \\
\text { improved health }\end{array}$} & 217 (98.64) & $171(99.42)$ & $46(95.83)$ & \multirow[t]{2}{*}{$0.990^{*}$} \\
\hline & Mean (SD) & Mean (SD) & Mean (SD) & \\
\hline BMI index - kg/m $\square$ & $19.02 \pm 0.99$ & $19.13 \pm 1.01$ & $18.62 \pm 0.82$ & $<0.001^{* *}$ \\
\hline Haller index on chest X-ray & $2.45 \pm 0.21$ & $2.46 \pm 0.23$ & $2.41 \pm 0.14$ & $0.067^{* *}$ \\
\hline
\end{tabular}

BMI: body mass index; SD: standard deviation; ${ }^{*}$ Chi-square test, ${ }^{* *}$ Mann-Whitney U test

\section{Discussion}


In resource-scare conditions such as Vietnam, it difficult us to acquire all important data for a large cohort of consecutive PE patients who underwent VATS for the NUSS procedure. Hence, we believe that current rare evidence drawn from this cohort enables us to give a real picture in clinical practice valuably not only for Vietnam but also for countries with similar resource-scare conditions. As is known in the literature, proper metal bar shape is crucial in the Nuss procedure for patients with PE. Kelly R. E et al. (2010) favored a gentle semicircular curve laterally with a 2 to $4 \mathrm{~cm}$ flat segment in the center to support the sternum [19]. Slight overcorrection of depression is preferred to undercorrection because the authors believed it minimizes the risk of recurrence and decreases the risk of cartilage buckling [19]. Park et al favor an asymmetrically bent bar for patients with asymmetric depression [20]. In this cohort, we used a ruler bent to the desired thoracic shape and then bent the metal bar to the shape and size of the ruler. We begin to bend the middle of the bar gradually to its ends; bars are usually taken to be 1 inch smaller compared to the distance between the two middle armpit lines. We have found that symmetrically bent bars tend to be unstable for the patients' chest.

In our series, most patients received only 1 bar $(n=350,95.89 \%)$, while 15 patients received 2 bars (4.11\%). The 2-bar insertion was indicated for most elderly patients with severe PE and wide concave area; no case was placed with 3 metal bars. Our finding was consistent with previous reports when both revealed that elderly patients need to put more metal bars compared to younger patients [21], [22]. The number of patients receiving 2 metal bars was lower in our study than in previous studies [21], [22]. For instance, in an early report by Pilegaard HK, 121 patients were inserted with 1 metal bar (66.8\%), 57 cases were inserted with 2 metal bars (32\%), and 2 cases were inserted with 3 metal bars (1.2\%) [22]. Of 1006 PE patients in Krystian Pawlak's recent series from July 2002 to September 2016, one bar was implanted in $452(44.9 \%)$ patients, two bars were inserted into $545(54.2 \%)$ patients, whilst three bars were inserted into $9(0.9 \%)$ patients [21]. The patient's chest wall became more solid after puberty and harder to bend; hence, the insertion of 2 metal bars was commonly shown with better and more stable results. Previous authors suggest that PE patients with Marfan syndrome or grand canyon PE and asymmetrical PE require two metal bars. The rate of using 2 metal bars has increased gradually in recent years; in particular, the insertion of 3 metal bars for PE patients has been reported in several studies. In the treatment of PE, the stability of the metal bar in the thorax is improved, and there is no need for re-surgical intervention when 2 metal rods are implanted in the first surgery. The use of two metal bars during the first treatment has become the standard of minimally invasive surgery for PE [1], [23], [24].

Previous reports have shown the safety of the thoracoscopic Nuss procedure with a decreased frequency of serious intraoperative and postoperative complications, especially in patients with complex PE who experienced a previous history of thoracic surgery and recurrent PE [18], [10], [12]. Usually, the surgeon stands to the right of the patient using the supportive thoracoscopic approach, which is consistent with the classic Nuss procedure that chooses the entrance from the right chest wall. The VATS technique was acquired for all PE patients in this cohort, and $\mathrm{CO} 2$ insufflation was used to create an artificial pneumothorax and thereby increase the empty pleural space. Forty-three patients underwent right VATS (11.78\%), while left VATS was performed in 308 patients (84.38\%). In particular, a bilateral thoracoscopic approach was applied in 14 PE patients (3.84\%) due to severe PE compressing the lungs and heart and 
failed Ravitch procedure. In the early years of implementing VATS in the Nuss procedure, we applied both thoracoscopic approaches, including the right chest wall and the left chest wall. Nevertheless, during recent years, most of the cases were approached through left thoracoscopy in our institution. With the left thoracoscopic approach, we found that the surgical field of view was wide enough to clearly observe the entire pleural cavity, left lung, blood vessel, heart, pericardium, mediastinum and diaphragm; hence, serious complications can be avoided. Several authors favored placing the trocar in the middle axillary line [14], [25]. However, we found that the trocar placed in the middle axillary line can result in vision that can be hindered by the heart, lungs, and diaphragm, especially in cases of asymmetrical severe PE. We agree with Hendrickson et al. and Palmer et al. that left thoracoscopy has an advantage over right thoracoscopy and does not increase the risk of heart injury [11], [13]. Hemostasis is well controlled, and lung damage is avoided under endoscopic observation. During the process of ballooning through the endotracheal tube to enlarge the bilateral lungs, we clearly observed that the two lungs were enlarged and until the lungs were maximized; when the pleural space was empty, we withdrew the endoscopic trocar. Therefore, a pleural drainage catheter was not placed during surgery.

During the surgery, no serious complications or fatalities were observed in our series. Early postoperative complications were recorded up to 30 days during the hospital stay, with the most frequent pneumothorax occurring in 5 patients (1.37\%). In most of the studies, the most common early complication was pneumothorax, and its occurrence frequency was estimated to be from $1 \%$ to $64 \%$ [12], [26], [27], [28]; however, in the majority of cases, the pneumothorax spontaneously resolved, and only a small number of patients required surgical treatment. In addition, no patient with postoperative bleeding required a blood transfusion, and no other serious complications or deaths were recorded in our study. Late postoperative complications are events that occur when the patient is discharged from the hospital, such as metal bar deviation, metal bar allergy, or surgical wound infection. Regarding late postoperative complications, we recorded surgical wound infection in 2 patients $(0.55 \%)$, metal bar deviation in 5 patients $(1.37 \%)$, metal bar allergy in 10 patients $(2.74 \%)$, and recurrent PE in $2(0.55 \%)$. Importantly, no mortality was reported in this series. Bar displacement is one of the most common complications [29]. According to the literature, the incidence of bar displacement was $3-20 \%$, and this incidence was lower in our study (1.37\%) [20], [18], [30].

Metal bar allergy occurred in $2.74 \%$ of our patients $(n=10)$ with manifestations such as mild fever, inflammation, wound fluid buildup, blisters, and erythema. Our study had a similar proportion of metal bar allergies compared to others, such as Nuss D. et al. (2008) of 2.9\% [31], Kelly R. E et al. (2010) of 2.8\% [19]. In particular, we found that 6 out of 10 patients with metal bar allergies had a history of allergic diseases such as allergic rhinitis, drug allergies, weather allergies, food allergies, and asthma. This helps us gain more experience in the prognosis, diagnosis, and treatment of allergy drugs as well as closely monitor allergy status after surgery for appropriate management measures.

The Nuss procedure is known as a prepared clean surgery. Although very few reported wound infections have occurred, infections are extremely serious because foreign material has been placed inside the body. In our study, of 365 postoperative patients, there were 2 cases of late wound infection $(0.55 \%)$. Those 
patients received prompt antibiotic treatment and were discharged after 7-10 days of treatment. There were no cases of deep infection of the metal bar. Some studies in the literature showed that the incidence of late wound infection was 1.5\% [32], [33]. In addition, other complications should also be noted in our report. Five patients had chest wall pain lasting at 1 month and 2 months postoperatively, were taken oral pain relievers and performed breathing exercises; their pain then subsided and disappeared. There were 2 patients with recurrent PE and 5 with persistent PE after the bar removal operation.

We recorded 124 out of 365 patients with mid-term results at $6-30$ months postoperatively. Of 124 patients, most patients had unremoved bars $(n=121,97.58 \%)$, and only 3 patients had removed bars $(2.42 \%)$. Most patients had body weight gain $(n=97,78.23 \%)$, increased physical activity and improved health $(n=115,92.74 \%)$. The mean BMI index was $18.77 \pm 0.92 \mathrm{~kg} / \mathrm{m}^{2}$ in 124 patients. The Haller index, which is the ratio of the widest transverse $(T)$ diameter of the chest to the shortest distance between the anterior spine and posterior sternum, has been used to quantify the severity of PE. Additionally, the size of the PE was postoperatively evaluated according to the Haller index based on two projection chest $X$-rays - postero-anterior and right lateral. At 6 - 30 months postoperatively, we measured the mean Haller index of $2.44 \pm 0.15$. The Haller index is, however, of limited value because it does not accurately reflect threedimensional morphological changes in the thoracic cavity before and after the Nuss procedure. In midterm results, we found that the patients receiving bar removal had a significantly higher mean BMI index compared to those not yet receiving bar removal $(20.30 \pm 1.11$ vs $18.73 \pm 0.89)(p<0.05)$. The proportion of patients with increased physical activity and improved health following surgery differed significantly between those receiving bar removal $(n=43,100.00 \%)$ and those not yet receiving bar removal $(n=112$, $92.56 \%)(p<0.05)$. To evaluate postoperative long-term results at over 30 months, 220 out of 365 patients $(60.27 \%)$ were included in this study. Of those, 172 received bar removal $(78.18 \%)$, and 48 had not yet received bar removal (21.82\%). A total of 217 patients (98.64\%) had both body weight gain, increased physical activity and improved health. In 220 patients over 30 months postoperatively, the mean BMI index was $19.02 \pm 0.99 \mathrm{~kg} / \mathrm{m}^{2}$, and the Haller index on chest X-ray was $2.45 \pm 0.21$. As was reported in our study, at over 30 months postoperatively, the BMI index was significantly higher in patients receiving bar removal than in those not yet receiving bar removal $(19.13 \pm 1.01$ vs $18.62 \pm 0.82)(p<0.05)$.

Several limitations need to be noted in this study. It was a retrospective one-center experience. Although $60.27 \%$ of patients were followed up over 30 months, mid-term to long-term postoperative outcomes after removal of bar(s) should be investigated in detail in a further study. In particular, metal support is still in place in a number of PE patients, not enabling us to have the complete evaluation of postoperative results.

\section{Conclusions}

In conclusion, the VATS technique in the Nuss procedure for PE was a safe and effective approach that minimized the occurrence of serious intra- and postoperative complications. From our initial oneinstitution experience in a resource-scare country, good mid-term to long-term postoperative outcomes in PE patients were obtained with VATS treatment using the minimally invasive Nuss method. Current rare 
evidence drawn from this cohort enables us to provide a real picture of the application, modification and development of VATS in the Nuss procedure not only for Vietnam but also for countries with similar resource-scarce conditions.

\section{Declarations}

\section{Ethical Approval}

All procedures conducted in studies involving human subjects were in compliance with the ethical principles of the Institutional and/or National Study Committee and the Helsinki Declaration of 1964 and its corresponding amendments or equivalent ethical standards. This study was approved by the Ethics Board of Hanoi Medical University. All individual participants included in the study obtained informed consent.

Competing Interests: The authors declare no competing interests.

\section{References}

1. Nuss, D., et al., A 10-year review of a minimally invasive technique for the correction of pectus excavatum. Journal of pediatric surgery, 1998. 33(4): p. 545-552.

2. Coln, D., et al., Early experience with the Nuss minimally invasive correction of pectus excavatum in adults. World journal of surgery, 2002. 26(10): p. 1217-1221.

3. Kelly Jr, R.E., et al., Prospective multicenter study of surgical correction of pectus excavatum: design, perioperative complications, pain, and baseline pulmonary function facilitated by internet-based data collection. Journal of the American College of Surgeons, 2007. 205(2): p. 205-216.

4. Antonoff, M.B., et al., Retrospective review of reoperative pectus excavatum repairs. Journal of pediatric surgery, 2010. 45(1): p. 200-205.

5. Croitoru, D.P., et al., The minimally invasive Nuss technique for recurrent or failed pectus excavatum repair in 50 patients. Journal of pediatric surgery, 2005. 40(1): p. 181-187.

6. Miller, K., et al., Minimally invasive bar repair for 'redo'correction of pectus excavatum. Journal of pediatric surgery, 2002. 37(7): p. 1090-1092.

7. Redlinger Jr, R.E., et al., One hundred patients with recurrent pectus excavatum repaired via the minimally invasive Nuss technique-effective in most regardless of initial operative approach. Journal of Pediatric Surgery, 2011. 46(6): p. 1177-1181.

8. Schaarschmidt, K., et al., Extrapleural, submuscular bars placed by bilateral thoracoscopy-a new improvement in modified Nuss funnel chest repair. Journal of pediatric surgery, 2005. 40(9): p. 14071410.

9. Takagi, S., et al., A new sternum elevator reduces severe complications during minimally invasive repair of the pectus excavatum. Pediatric surgery international, 2012. 28(6): p. 623-626. 
10. Bufo, A.J. and M.M. Stone, Addition of thoracoscopy to Nuss pectus excavatum repair. Pediatric Endosurgery and Innovative Techniques, 2001. 5(2): p. 159-162.

11. Hendrickson, R.J., et al., Efficacy of left thoracoscopy and blunt mediastinal dissection during the Nuss procedure for pectus excavatum. Journal of pediatric surgery, 2005. 40(8): p. 1312-1314.

12. Cheng, Y.-L., et al., Efficacy and safety of modified bilateral thoracoscopy-assisted Nuss procedure in adult patients with pectus excavatum. European journal of cardio-thoracic surgery, 2008. 34(5): p. 1057-1061.

13. Palmer, B., S. Yedlin, and S. Kim, Decreased risk of complications with bilateral thoracoscopy and left-to-right mediastinal dissection during minimally invasive repair of pectus excavatum. European journal of pediatric surgery, 2007. 17(02): p. 81-83.

14. Saxena, A.K., C. Castellani, and M.E. Höllwarth, Surgical aspects of thoracoscopy and efficacy of right thoracoscopy in minimally invasive repair of pectus excavatum. The Journal of Thoracic and Cardiovascular Surgery, 2007. 133(5): p. 1201-1205.

15. Gips, H., K. Zaitsev, and J. Hiss, Cardiac perforation by a pectus bar after surgical correction of pectus excavatum: case report and review of the literature. Pediatric surgery international, 2008. 24(5): p. 617-620.

16. Zhang, D.-K., et al., Surgical correction of 639 pectus excavatum cases via the Nuss procedure. Journal of thoracic disease, 2015. 7(9): p. 1595.

17. Schaarschmidt, K., et al., Lessons learned from lethal cardiac injury by Nuss repair of pectus excavatum in a 16-year-old. The Annals of Thoracic Surgery, 2013. 95(5): p. 1793-1795.

18. Park, H.J., et al., The Nuss procedure for pectus excavatum: evolution of techniques and early results on 322 patients. The Annals of thoracic surgery, 2004. 77(1): p. 289-295.

19. Kelly, R.E., et al., Twenty-one years of experience with minimally invasive repair of pectus excavatum by the Nuss procedure in 1215 patients. Annals of surgery, 2010. 252(6): p. 1072-1081.

20. Park, H.J., S.Y. Lee, and C.S. Lee, Complications associated with the Nuss procedure: analysis of risk factors and suggested measures for prevention of complications. Journal of pediatric surgery, 2004. 39(3): p. 391-395.

21. Pawlak, K., et al., Video-assisted-thoracoscopic surgery in left-to-right Nuss procedure for pectus excavatum for prevention of serious complications-technical aspects based on 1006 patients. Videosurgery and Other Miniinvasive Techniques, 2018. 13(1): p. 95.

22. Pilegaard, H.K. and P.B. Licht, Routine use of minimally invasive surgery for pectus excavatum in adults. The Annals of thoracic surgery, 2008. 86(3): p. 952-956.

23. Pilegaard, H.K., Extending the use of Nuss procedure in patients older than 30 years. European journal of cardio-thoracic surgery, 2011. 40(2): p. 334-337.

24. Stanfill, A.B., et al., Nuss procedure: decrease in bar movement requiring reoperation with primary placement of two bars. Journal of Laparoendoscopic \& Advanced Surgical Techniques, 2012. 22(4): p. 412-415. 
25. Nuss, D., R.J. Obermeyer, and R.E. Kelly, Nuss bar procedure: past, present and future. Annals of cardiothoracic surgery, 2016. 5(5): p. 422.

26. Nuss, D., et al., Review and discussion of the complications of minimally invasive pectus excavatum repair. European journal of pediatric surgery, 2002. 12(04): p. 230-234.

27. Peter, S.D.S., et al., Use of a subxiphoid incision for pectus bar placement in the repair of pectus excavatum. Journal of pediatric surgery, 2010. 45(6): p. 1361-1364.

28. Castellani, C., et al., Early complications of the Nuss procedure for pectus excavatum: a prospective study. Pediatric surgery international, 2008. 24(6): p. 659-666.

29. Hebra, A., et al., Outcome analysis of minimally invasive repair of pectus excavatum: review of 251 cases. Journal of pediatric surgery, 2000. 35(2): p. 252-258.

30. Croitoru, D.P., et al., Experience and modification update for the minimally invasive Nuss technique for pectus excavatum repair in 303 patients. Journal of pediatric surgery, 2002. 37(3): p. 437-445.

31. Nuss, D. and R.E. Kelly, Minimally invasive surgical correction of chest wall deformities in children (Nuss procedure). Advances in pediatrics, 2008. 55(1): p. 395-410.

32. Shin, S., et al., Infectious complications after the Nuss repair in a series of 863 patients. Journal of pediatric surgery, 2007. 42(1): p. 87-92.

33. Goretsky, M.J. and M.M. McGuire. Complications associated with the minimally invasive repair of pectus excavatum. in Seminars in pediatric surgery. 2018. Elsevier.

\section{Figures}




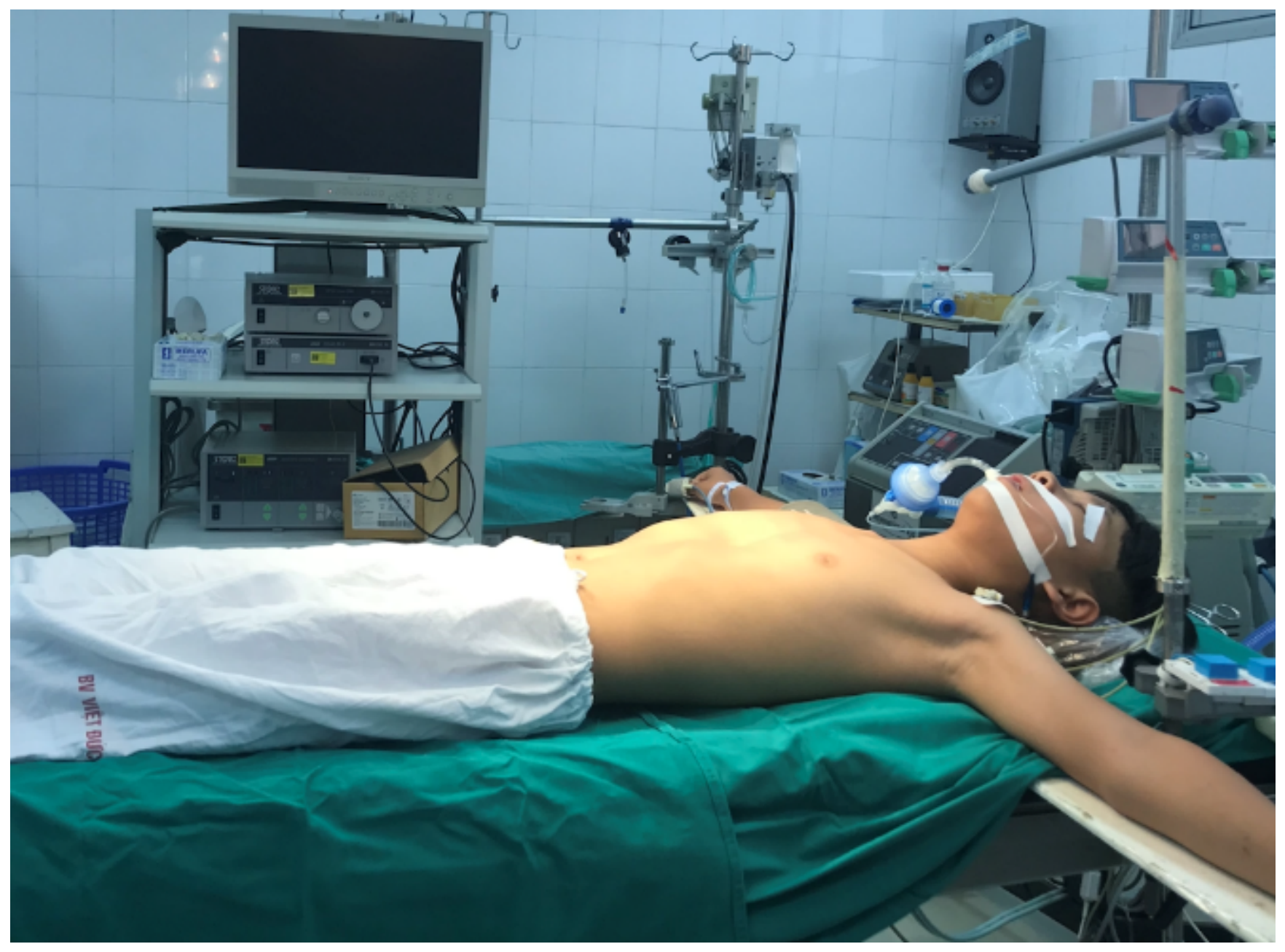

Figure 1

The supine position after general anesthesia 


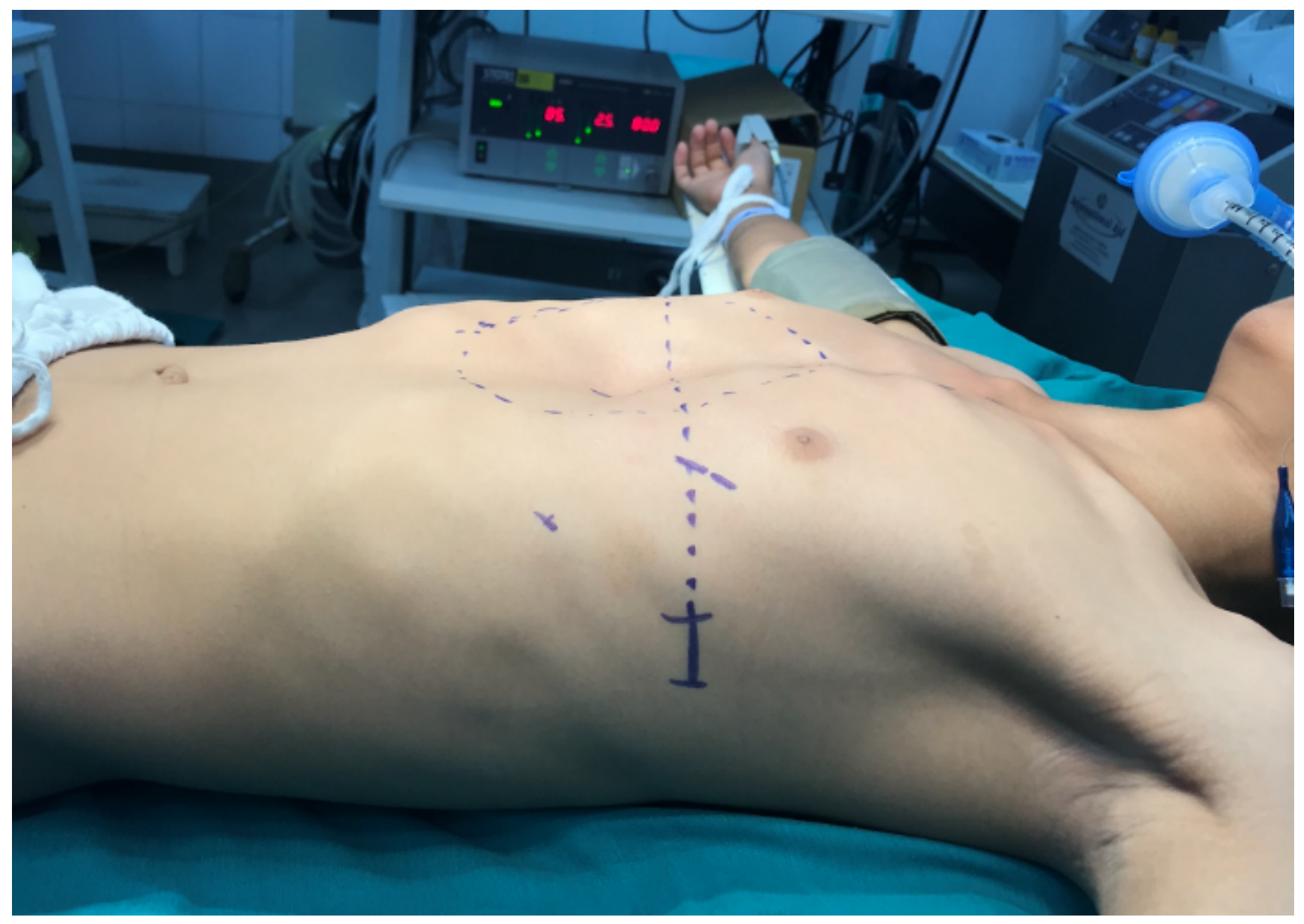

Figure 2

The determination of anatomic landmarks in surgery

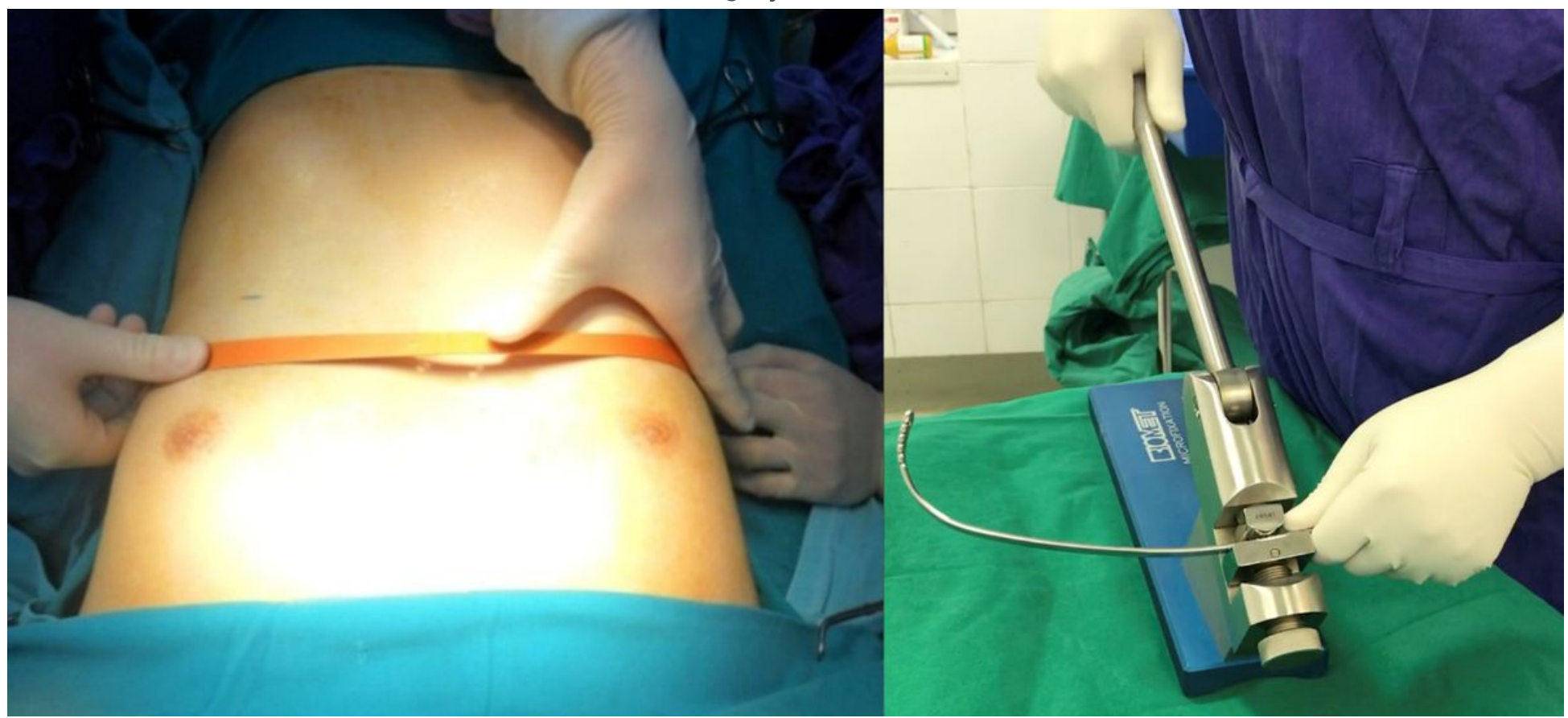

Figure 3 
(Left) a.The measurements of the thorax. (Right) b. The bending of the metal bar

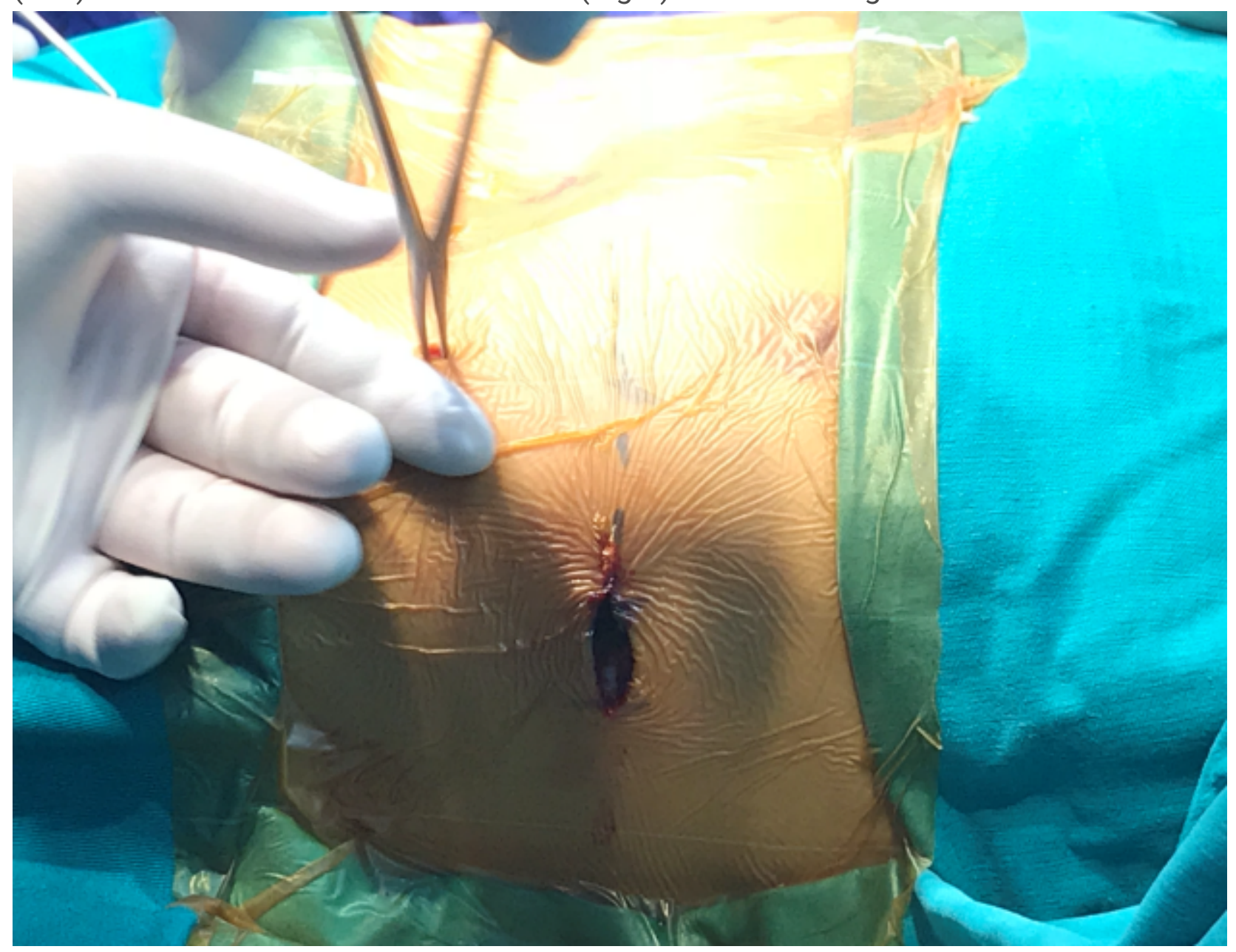

Figure 4

Skin incision position 


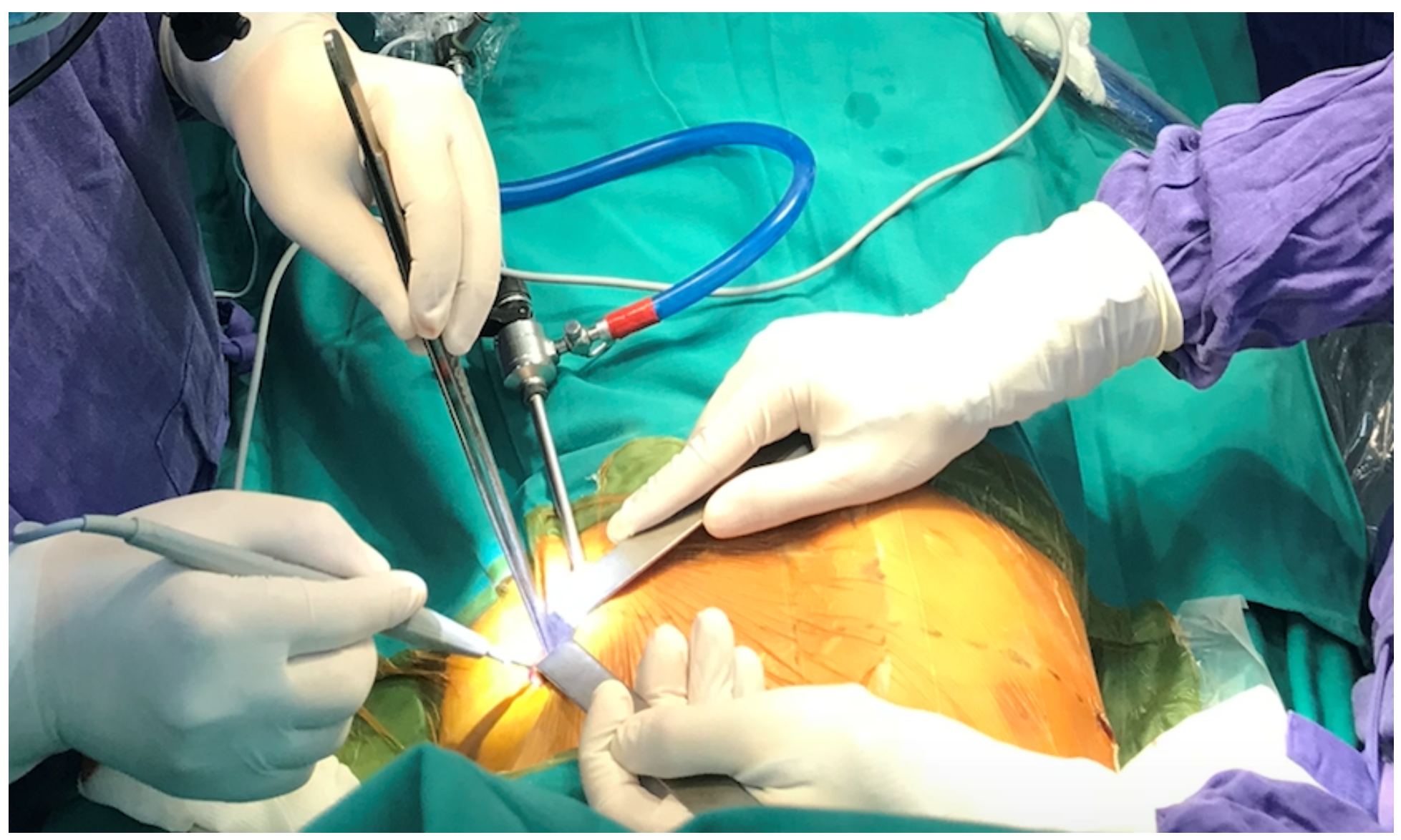

Figure 5

The creation of skin tunnel in the left chest wall

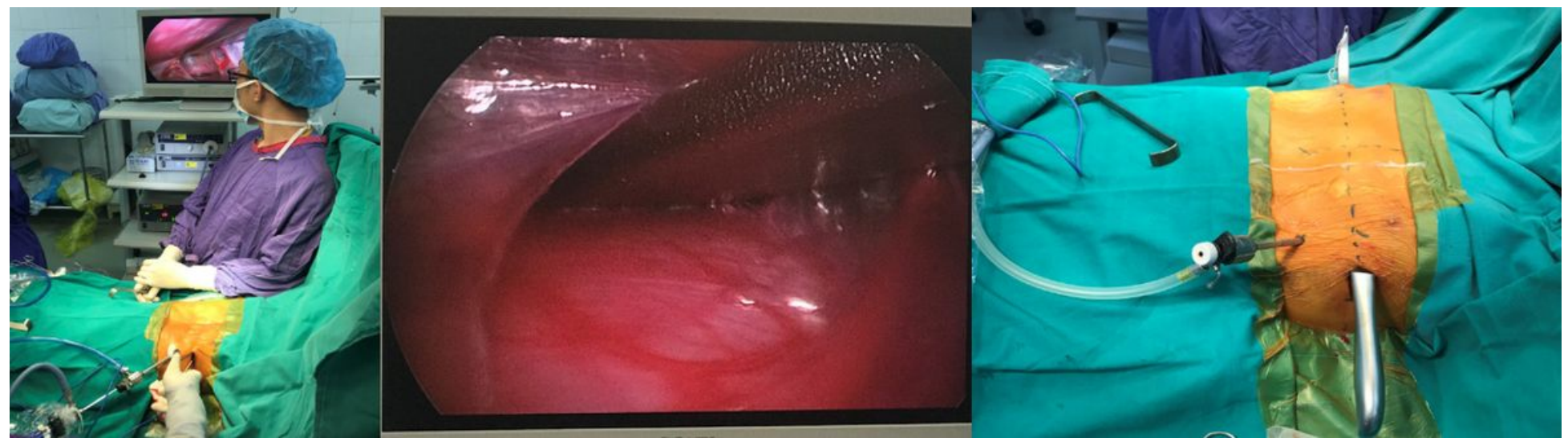

Figure 6

The process of created tunnel through the mediastinum under the control of the thoracoscopy 


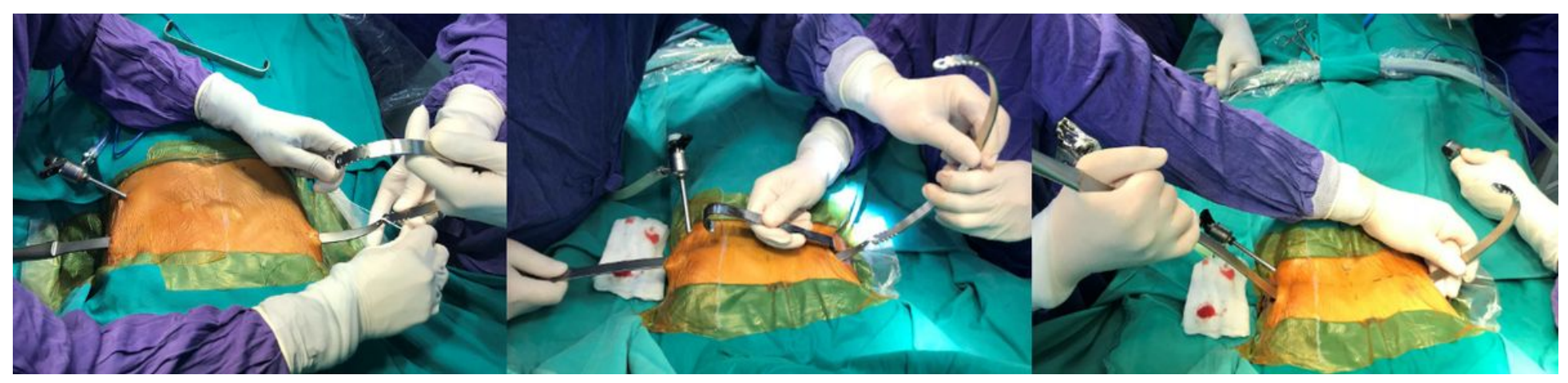

Figure 7

The process of created tunnel through the mediastinum under the control of the thoracoscopy

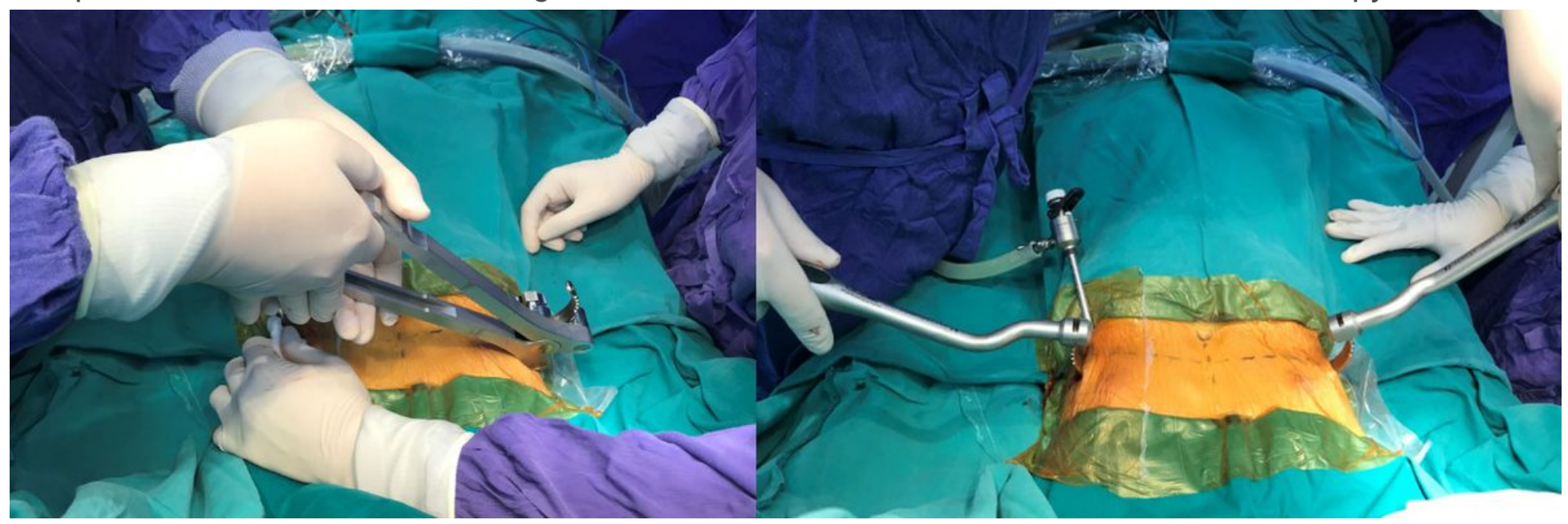

Figure 8

Bending and rotating the metal bar

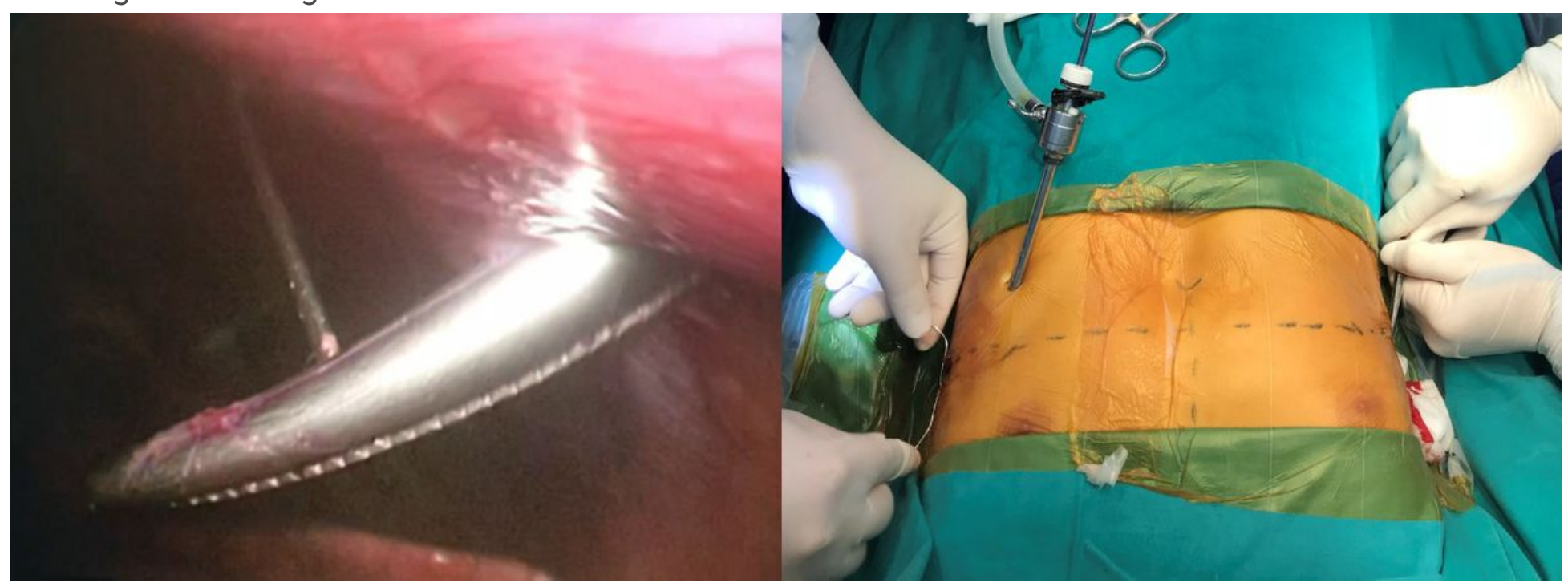

Figure 9

The pectus bar was anchored into the position by steel thread 